\title{
ABUNDANCIA Y DEPREDACIÓN DE Ceraeochrysa valida (NEUROPTERA: CHRYSOPIDAE) SOBRE Diaphorina citri (HEMIPTERA: LIVIIDAE) EN COLIMA, MÉXICO
}

\author{
ABUNDANCE AND PREDATION OF Ceraeochrysa valida (NEUROPTERA: \\ CHRYSOPIDAE) ON Diaphorina citri (HEMIPTERA: LIVIIDAE) IN COLIMA, \\ MEXICO
}

Martín Palomares-Pérez ${ }^{1}$, Miguel Angel Ayala-Zermeño ${ }^{1}$, Beatriz Rodríguez-Vélez ${ }^{1 *}$, José de Jesús de la Cruz-Llanas ${ }^{1}$, Jorge Antonio Sánchez-González ${ }^{1}$, Hugo César Arredondo-Bernal ${ }^{1}$ y Esther Gisela Córdoba-Urtiz ${ }^{1}$

\footnotetext{
${ }^{1}$ Centro Nacional de Referencia de Control Biológico, SENASICA-DGSV Km 1.5 Carretera TecománEstación FFCC, Colonia Tepeyac, Colima, México. C.P. 28110. mpalomares@colpos.mx, ayalazermeno@yahoo.com.mx, ‘beatriz_rv@yahoo.com, llanas_11@hotmail. com, j_asg2@hotmail.com, hcesar_64@yahoo.com.mx, gisela_1704@hotmail.com

* Autor para correspondencia E-mail: beatriz_rv@yahoo.com
}

\section{RESUMEN}

Diaphorina citri Kuwayama es considerada la plaga más importante de los cítricos por transmitir la bacteria Candidatus Liberibacter spp., causante de la muerte de millones de árboles en el mundo. Para recomendar al depredador Ceraeochrysa valida (Banks) en el control de este insecto se realizaron cuatro ensayos: 1) distribución y presencia de $C$. valida en huertas de limón en el estado de Colima; 2) máxima capacidad de depredación de $C$. valida sobre ninfas de $D$. citri bajo condiciones controladas; 3 ) tablas de vida de $D$. citri con la liberación de $C$. valida en condiciones naturales; y 4) depredación en campo de $C$. valida sobre $D$. citri. Los resultados fueron: Ceraeochrysa valida se presentó en todas las huertas evaluadas durante los doce meses del año. El máximo consumo promedio en laboratorio fue $258,26 \pm 3,80$ ninfas. La liberación de 20 larvas en campo dio una mortalidad de $160,29 \pm 26,32$ ninfas de $D$. citri contra $27,33 \pm 3,39$ sin liberación $(F=11,98, \operatorname{Pr}>0,0134)$. La mortalidad real de huevo y ninfa fue mayor con liberación de $C$. valida $(0,9768 ; 0,8482 ; 1,0000 ; 0,9922)$ que sin liberación $(0,8481 ; 0,4467 ; 1 ; 0,8032)$. El estimativo $R_{0}$ estuvo por debajo de la unidad con liberación $(0,1628 ; 0,5893 ; 0,000 ; 0,4870)$ y cerca de la unidad o arriba de ella sin liberación $(0,7278 ; 1,1733 ; 0,1231$; 1,0787). De acuerdo a los resultados $C$. valida podría ser considerado en el control biológico de $D$. citri.

Palabras clave: Control biológico, psílido asiático de los cítricos, tablas de vida, Huanglongbing (HLB), depredador.

\section{ABSTRACT}

Diaphorina citri Kuwayama is considered the most important pest of citrus since it transmits the bacterium Candidatus Liberibacter spp., causing the death of millions of citrus trees all over the world. This research studied the ability of Ceraeochrysa valida (Banks) as a biological control agent against $D$. citri. The study was conducted under field and laboratory conditions, considering four 
different criteria: 1) distribution and presence of $C$. valida in lemon orchards in the state of Colima, 2) maximum predation of $C$. valida on $D$. citri nymphs under laboratory conditions, 3) life tables of $D$. citri with release of $C$. valida under field conditions, and 4) predation of $C$. valida on $D$. citri in the field. The results showed that Ceraeochrysa valida was present in the orchards evaluated throughout the year. The average amount of $D$. citri nymphs consumed by $C$. valida under laboratory condition was $258.26 \pm 3.80$. The release of 20 larvae of $C$. valida in the field produced an average mortality of $160.29 \pm 26.32$ nymphs of $D$. citri against $27.33 \pm 3.39$ without release $(F=11.98, \operatorname{Pr}>F=0.0134)$. The analysis of life tables for the months of December, January, February and March showed that egg and nymph mortality was higher in treatments in which $C$. valida was released $(0.9768 ; 0.8482 ; 1$; $0.9922)$ compared to those in which no release occurred $(0.8481 ; 0.4467 ; 1 ; 0.8032)$. $R_{\mathrm{o}}$ reached values lower than one under release treatments $(0.1628 ; 0.5893 ; 0.000 ; 0.4870)$, while these were close to or higher than one $(0.7278 ; 1.1733 ; 0.1231 ; 1.0787)$ in the treatments without release. It can be concluded that $C$. valid can be considered as a biological control agent against $D$. citri.

Key words: biological control, Asian citrus psyllid, life tables, Huanglongbing (HLB), predator.

\section{INTRODUCCIÓN}

Diaphorina citri Kuwayama (Hemiptera: Liviidae), el psílido asiático de los cítricos (PAC), es una plaga y vector de la enfermedad de los cítricos conocida como Huanglongbing (HLB), considerada la más devastadora para este cultivo a nivel mundial (Halbert y Manjunath, 2004). Este insecto se desarrolla exclusivamente en plantas de la familia Rutaceae, particularmente del género Citrus y Murraya (Etienne et al., 1998). Diaphorina citri es de origen asiático y se reportó por primera vez en Campeche, México, en 2002 (Thomas, 2002); a partir de ese año su dispersión ha sido paulatina y actualmente está ampliamente distribuida en todas las áreas citrícolas del país (SCOPE, 2015). Las ninfas y los adultos se alimentan de la planta, succionan la savia de los brotes, y la mielecilla excretada provoca la aparición de fumagina (Halbert y Manjunath, 2004). Por su parte, el HLB fue detectado en México por vez primera en julio de 2009 en el estado de Yucatán (SAGARPA, 2009); actualmente está presente en 19 entidades citrícolas de México (SINAVEF, 2013) y su combate es una de las prioridades nacionales, ya que causa pérdidas económicas cuantiosas (Hodges et al., 2014). Los daños causados por la enfermedad están asociados con la disminución de la producción, la deformación y desarrollo de frutos, así como la muerte del árbol en un periodo de 5 a 8 años después que es infectado (Tsai et al., 2002; Halbert y Manjunath, 2004). A la fecha se desarrolla un programa de control biológico de su vector $D$. citri, mediante la liberación masiva del parasitoide Tamarixia radiata Burks (Hymenoptera: Eulophidae) y algunos hongos entomopatógenos como Isaria javanica (Bally) Samson y Hywel-Jones y Metarhizium anisopliae sensu lato (Metschnikoff) Sorokin (Ayala-Zermeño et al., 2015); de igual forma se están buscando alternativas que complementen los organismos benéficos incluidos en el programa de control de D. citri. Una de las opciones son los depredadores, entre los que destacan miembros del género Ceraeochrysa (Neuroptera: Chrysopidae), el cual abarca numerosas especies que tienen un gran potencial para ser utilizados en el control biológico de plagas agrícolas, además de que existe la tecnología para su reproducción masiva (Tauber et al., 2000). Algunos trabajos técnicos (López-Arroyo et al., 2005; Cortez-Mondaca et al., 2011) mencionan a la especie Ceraeochrysa valida (Banks) depredando a D. citri en las principales zonas productoras de cítricos en México. Sin embargo no hay trabajos formales bajo condiciones naturales que permitan conocer el potencial de este organismo en el control de D. citri. Por tal motivo, el presente estudio tuvo como objetivo evaluar la abundancia, distribución y la efectividad depredadora de $C$. valida tanto en campo como en laboratorio con la finalidad de conocer su potencial como agente de control biológico de D. citri en México.

\section{MATERIALES Y MÉTODOS}

El estudio se llevó a cabo mediante cuatro ensayos: 1) distribución y presencia de $C$. valida en huertas de limón en el estado de Colima; 2) máxima capacidad de depredación de C. valida sobre ninfas de $D$. citri bajo condiciones controladas; 3) tablas de vida de $D$. citri con la liberación de larvas de $C$. valida en condiciones naturales; y 4) depredación en campo de $C$. valida sobre $D$. citri.

1) Distribución y presencia de $C$. valida en huertas de limón en el estado de Colima

Se realizaron muestreos aleatorios mensualmente en huertas de cítricos en los municipios

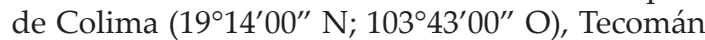
$\left(18^{\circ} 55^{\prime} 00^{\prime \prime} \mathrm{N} ; 103^{\circ} 53^{\prime} 00^{\prime \prime} \mathrm{O}\right)$, Armería $\left(18^{\circ} 56^{\prime} 00^{\prime \prime}\right.$ $\left.\mathrm{N} ; 103^{\circ} 58^{\prime} 10^{\prime \prime} \mathrm{O}\right)$ e Ixtlahuacán $\left(19^{\circ} 00^{\prime} 00^{\prime \prime} \mathrm{N}\right.$; $103^{\circ} 45^{\prime} 00^{\prime \prime}$ O) de enero a diciembre de 2014. Los huertos mostraron la presencia de $D$. citri. Para 
cada punto de muestreo se tomaron las coordenadas geográficas con el sistema de posicionamiento global (GPS 12 XL Garmin Olathe, KS, USA). Se colectaron adultos en 4 huertas de cada municipio. La recolecta se realizó en diez árboles utilizando una red de golpeo mediante diez golpes sobre la periferia del árbol. El material biológico se trasladó al Laboratorio del Departamento de Insectos Entomófagos (DIE) del Centro Nacional de Referencia de Control Biológico (CNRCB) en Tecomán, Colima, México.

La identificación de los ejemplares se realizó mediante caracteres morfológicos externos e internos, con la ayuda de microscopios estereoscópicos y compuestos utilizando las claves taxonómicas de Brooks y Barnard (1990) para género y la descripción de Tjeder $(1966 ; 1971)$ revisada por Barnard (1984) para la interpretación de genitalia de machos y hembras. Para la identificación de especies se utilizaron las claves propuestas para Chrysoperla (Brooks, 1994) y Ceraeochrysa (Tauber et al., 2000).

2) Máxima capacidad de depredación de $C$. valida sobre ninfas de $D$. citri bajo condiciones controladas

El experimento se realizó en el laboratorio del DIE del CNRCB bajo condiciones de $25 \pm 2^{\circ} \mathrm{C}$, HR de $60-70 \%$ y fotoperiodo de $12 \mathrm{~h}$ luz. Los psílidos y crisópidos fueron proporcionados por el DIE del CNRCB.

Antes del inicio de cada experimento se aseguró la edad de los estadios larvarios de $C$. valida; la larva I (LI) fue aquella que eclosionó del huevo, la larva II y III son las que presentaron menos de $24 \mathrm{~h}$ de haber mudado del estadio anterior inmediato. Las larvas se alimentaron con huevos de Sitotroga cerealella (Oliver) hasta $24 \mathrm{~h}$ antes de iniciar el ensayo.

Para medir la capacidad máxima de depredación de cada estadio larvario de C. valida se co- locaron 300 ninfas de D. citri (60 ninfas de cada uno de los cinco estadios ninfales) en cajas de Petri de plástico de $5,5 \mathrm{~cm}$ de diámetro con un disco de limón Persa en su base [Citrus aurantifolia (Christm.) Swingle] como alimento para las ninfas, posteriormente se colocó por separado, un individuo de cada uno de los estadios larvarios de C. valida, por un periodo de $24 \mathrm{~h}$, determinando durante este tiempo, el número de ninfas consumidas. Se realizaron 10 repeticiones por estadio larval y se mantuvieron en una cámara de cría bajo las condiciones antes mencionadas.

El consumo de presa entre los tres estadios larvales fue analizado con una prueba de ANOVA y en caso de observar diferencias significativas se aplicó una prueba de comparación múltiple de medias de Tukey $(\alpha=0,05)$ (SAS, 2008).

3) Tablas de vida de $D$. citri con liberación de larvas de $C$. valida en condiciones naturales

Para contrastar los impactos de las liberaciones de $C$. valida sobre las condiciones naturales de supervivencia de $D$. citri, se construyeron durante los meses de diciembre de 2014 a marzo de 2015 cuatro tablas de vida de $D$. citri, estableciéndose para cada intervalo de edad los parámetros siguientes:

$1_{x}=$ Individuos vivos entre $1_{x}$ y $1_{x}+1 ; d_{x}=N u ́-$ mero de muertos entre edades $1_{x}$ y $1_{x}+1 ; q_{x}=$ Tasa de mortalidad $\left(\mathrm{d}_{x} / 1_{\mathrm{x}}\right)$; $\mathrm{k}$-value $=$ Fuerza de mortalidad; $\mathrm{R}_{\mathrm{o}}=$ Tasa neta de reproducción $\mathrm{l}_{\mathrm{x}} \mathrm{m}_{\mathrm{x}}$

Las tablas de vida fueron analizadas mediante cohortes establecidas en árboles de limón mexicano bajo condiciones naturales (Tabla 1). La huerta donde se desarrolló el experimento (18 $56^{\prime} 32,88^{\prime \prime}$ $\left.\mathrm{N} ; 103^{\circ} 56^{\prime} 05.18^{\prime \prime} \mathrm{O}\right)$ se caracterizó por presentar árboles de 10 años de edad en promedio y un manejo de cinco años sin aplicación de plaguicidas.

Los cohortes se formaron recolectando en la

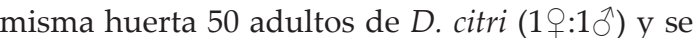
colocaron respectivamente durante un periodo

Tabla 1. Condiciones meteorológicas presentes en la evaluación de tablas de vida de Diaphorina citri con y sin liberación de Ceraeochrysa valida

Table 1. Weather conditions in assessing life tables of Diaphorina citri with and without release of Ceraeochrysa valida.

\begin{tabular}{lrcccc}
\hline Fecha & Prec. & T. Máx. & T. Mín. & T. Med. & HR \\
\hline 2014-2015 & $\mathrm{mm}$ & ${ }^{\circ} \mathrm{C}$ & ${ }^{\circ} \mathrm{C}$ & ${ }^{\circ} \mathrm{C}$ & $\%$ \\
Diciembre & 39,40 & 31,05 & 19,32 & 24,38 & 86,28 \\
Enero & 0,00 & 31,69 & 18,97 & 24,71 & 82,02 \\
Febrero & 96,40 & 29,40 & 18,33 & 23,34 & 87,14 \\
Marzo & 146,20 & 29,88 & 18,16 & 23.76 & 83,95 \\
\hline
\end{tabular}

Prec.: Precipitación; T. Máx.: Temperatura máxima; T. Mín.: Temperatura mínima; T. Med.: Temperatura media; HR.: Humedad relativa.

Fuente: Estación Meteorológica de Armería, INIFAP (1854'52,7" N, 10358'9,2" O). 
de 48 h en un brote tierno y limpio de 5 a $10 \mathrm{~cm}$ de longitud para que ovipositaran. Para evitar la fuga de los insectos, el brote con los adultos fueron cubiertos con bolsas de tela de organza de 10 $x 5 \times 5 \mathrm{~cm}$.

Al transcurrir el tiempo establecido se extrajeron y sacrificaron los adultos, se retiró la tela de organza y se registró con la ayuda de una lupa 10X el número de huevos presentes en el brote. Los huevos y las ninfas que emergieron se expusieron en ambos tratamientos hasta completar su desarrollo a los factores naturales de mortalidad: tratamiento con liberación semanal de 20 larvas del segundo estadio de $C$. valida y tratamiento sin liberación.

Se construyeron para cada mes de evaluación, dos tablas de vida sin liberación y dos con liberación de $C$. valida, con intervalos de 15 días cada una. Para cada tabla de vida, el número de individuos de $D$. citri que sobrevivió y murió en cada etapa por cada una de los cohortes se combinaron mensualmente para su análisis. Las larvas del depredador utilizado en el tratamiento con liberación fueron facilitadas por el DIE del CNRCB.

Para el registro de las evaluaciones se asignaron primeramente cuatro categorías a $D$. citri; huevos, ninfa chica (estadio 1 y 2), ninfa grande (estadio 3, 4 y 5) y adulto. Apoyados con una lupa $10 \mathrm{X}$, se realizaron observaciones directas sobre el brote infestado y se registró la muerte de los individuos. Las evaluaciones se realizaron por un periodo de $30 \mathrm{~min}$, con dos observaciones diarias, por lo tanto, un total de 8 observaciones puntuales fueron hechas durante una evaluación de 4 $\mathrm{h}$ en intervalos de $24 \mathrm{~h}$ cada una. Se registró la ausencia del huevo o ninfa de $D$. citri y se identificó a nivel de especie o género los depredadores o parasitoides que se observaron con mayor frecuencia en una periferia de $40 \times 40 \mathrm{~cm}$ alrededor del brote infestado.

Los parámetros obtenidos se utilizaron para definir la mortalidad real (relación entre el número de individuos que muere en una etapa $\left(d_{x}\right)$ y el número inicial en la primer etapa de la tabla de vida), la mortalidad aparente (mortalidad que sucede en la población en ausencia del otro factor de mortalidad) (Bellows y Van Driesche, 1999), y la tasa neta de reproducción $\left(\mathrm{R}_{\mathrm{o}}\right)$, los cuales sirvieron para comparar el impacto de la liberación de C. valida.

Para asegurar la identificación de las especie de insectos que interfieren en el comportamiento de $C$. valida se realizaron muestreos previos sobre los árboles que contenían los brotes evaluados. La fauna benéfica y perjudicial se identificó con las claves correspondientes; para los coccinélidos se usaron las claves de Gordon (1985), para hemípteros las claves y artículos de Hodges y
Evans (2005), Peña-Martínez et al. (2002), Petrakis y Moulet (2011), Weirauch et al. (2012), para Hymenoptera las claves de Burks (2003), para Mantidae las claves de Florida Preying Mantids (2003). Mientras que la identificación de los aislados de hongos entomopatógenos se llevó a cabo con la ayuda de las claves taxonómicas de Barnett y Hunter (1998).

\section{4) Depredación en campo de $C$. valida sobre $D$. citri}

Veinte larvas del segundo estadio de C. valida fueron expuestas durante un periodo de $24 \mathrm{~h}$ en brotes infestados con los cinco estadios ninfales de D. citri, para determinar durante este periodo de tiempo el número de individuos consumidos. Se realizaron 4 repeticiones y se compararon contra cuatro brotes sin liberación. Con la ayuda de un lupa 10X se contabilizó previamente el número de ninfas presentes en los brotes y por diferencia se consideró la cantidad de individuos muertos.

El diseño experimental consistió en bloques completamente al azar analizados con una prueba de ANOVA. En caso de observar diferencias significativas se aplicó una prueba de comparación múltiple de medias de Tukey $(\alpha=0,05)$ (SAS, 2008).

\section{Resguardo de material biológico}

Los insectos identificados se encuentran en la Colección de Insectos Entomófagos (CIE) del CNRCB (CIE-CNRCB).

\section{RESULTADOS}

1) Distribución y presencia de C. valida en huertas de limón en el estado de Colima

Un total de 363 individuos de C. valida fueron colectados, y fue la segunda especie más abundante en los cuatro municipios durante los doce meses. A partir de febrero se identificó a Ceraeochrysa claveri (Navas) (310) y Ceraeochrysa cincta (Banks) (318) mientras que Chrysoperla externa (Hagen) fue la especie más abundante (860) y se presentó a partir de marzo.

2) Máxima capacidad de depredación de $C$. $v a$ lida sobre ninfas de $D$. citri bajo condiciones controladas

El consumo promedio de $C$. valida fue de 258,26 $\pm 3,80$ ninfas con un máximo de 280 y mínimo de 214 , sin registrar preferencia por algún instar en especial. Con un $\alpha=0,05$ se observan diferencias significativas en la alimentación de los tres estadios larvales ( $\mathrm{F}=8,79, \operatorname{Pr}>0,0012)$. Las pruebas de Tukey mostraron mayor capacidad de depredación de la LIII $(269 \pm 1,61$ a) y el LII $(262 \pm 5,00$ a), mientras que el LI $(245 \pm 4,81 b)$ es el estadio que consumió menor cantidad de ninfas. 


\section{3) Tablas de vida de D. citri con la liberación de C. valida en condiciones naturales}

En la zona de estudio se identificaron un total de nueve especies de depredadores [C. valida, C. cinta, C. claveri, Chr. externa, (Neuroptera: Chrysopidae); Olla v-nigrum (Mulsant), Cycloneda sanguinea L, Exochomus marginipennis (LeConte) (Coleoptera: Coccinellidae); Zellus renardii (Kolenati) (Hemiptera: Reduviidae); Mantis sp. (Mantodea: Mantidae) y el parasitoide (Tamarixia radiata (Waterston) (Hymenoptera: Eulophidae)]. Además se registró un hongo entomopatógeno, Hirsutella sp. y dos plagas agrícolas; Toxoptera sp. (Hemiptera: Aphididae) y Aleurocanthus sp. (Hemiptera: Aleyrodidae).

Las observaciones permitieron registrar el momento que $C$. valida realizó la depredación de $D$. citri en los tratamientos con su liberación. Se registró la ocurrencia de $C$. cincta y $C$. claveri durante las evaluaciones, así mismo se observó al coccinélido $C$. sanguinea alimentándose de $D$. citri en brotes sin liberación.

Las tablas de vida mostraron que la mortalidad real total de huevo y ninfa es mayor en los tratamientos con liberación de $C$. valida en tres de las cuatro tablas de vida (Tabla 2 y 3). En el mes de febrero (Tabla 3), la mortalidad real es igual en ambos tratamientos, debido a que en esta fecha hubo presencia de lluvia, factor importante de mortalidad de huevos y ninfas de $D$. citri.

Los factores naturales, la infertilidad de los huevos, la liberación de C. valida y la presencia de otros enemigos naturales dieron como resultado la mayor mortalidad real en el estado de huevo en las cuatro tablas de vida (Tabla 2 y 3 ).

La mortalidad real en el estado de ninfa fue mayor en los tratamientos con liberación de $C$. valida con excepción del mes de enero (Tabla 2), donde se observó parasitismo de $T$. radiata. De acuerdo a esto, la mortalidad aparente indicó que la mortandad de D. citri sería mayor en ausencia del parasitoide.

En las tablas de vida sin liberación de C. valida, el estimativo $\mathrm{R}_{\mathrm{o}}$ de los meses de diciembre, enero y marzo (Tabla 2 y 3 ) se encontró cerca o por arriba de la unidad, lo que implica un aumento de la población de $D$. citri, resultado contrario a los tratamientos con liberación, donde $\mathrm{R}_{\mathrm{o}}$ en los mismos meses evaluados (Tabla 2 y 3 ) está por debajo de la unidad, indicando que la población decreció con la liberación de C. valida. Para el mes de febrero el estimativo $R_{0}$ con y sin liberación (Tabla 3) estuvo debajo de la unidad, lo que implica que el comportamiento de la población en ambos tratamientos fue afectada por la lluvia presente durante la evaluación.
4) Depredación en campo de $C$. valida sobre $D$. citri

En la Tabla 4 se observa que la acción de los enemigos naturales y las condiciones naturales ejercen una mortalidad en D. citri de $23,01 \%$. La misma tabla nos indica que si se libera $C$. valida, esta mortalidad se incrementa a un $73,51 \%$.

Utilizando una transformación de datos a raíz cuadrada y con un $\alpha=0,05$, la liberación de $C$. valida sobre las ninfas de $D$. citri muestra efectos diferentes sobre brotes sin liberación $(\mathrm{F}=$ $11,98 ; \operatorname{Pr}>F=0,0134)$. Con la prueba de comparación múltiple de medias de Tukey se observó que la mayor mortalidad se presentó en el tratamiento con liberación de C. valida (liberación $160,29 \pm 26,32$ a; sin liberación $27,33 \pm 3,39$ b).

\section{DISCUSIÓN}

Ceraeochrysa valida es un depredador de la familia Chrysopidae que ha sido reportado desde el Ecuador, el Caribe, Centro América, México y Estados Unidos (Adams, 1982). En México se ha registrado en Baja California Sur, Tamaulipas, Yucatán, Nuevo León, Colima, Michoacán (Tauber y De León, 2001), Morelos (Valencia-Luna et al., 2006) y Sinaloa (Cortez-Mondaca et al., 2011). Además de estar presente en el cultivo de cítricos, existen reportes donde asocian a esta especie en plantaciones de algodón, papaya, maíz y hortalizas, donde las larvas probablemente se alimentan de áfidos y aleiródidos (Tauber et al., 2000).

Durante el presente estudio se identificó a $C$. valida como la segunda especie más abundante de crisópidos presentes en el área de estudio, de igual forma, Valencia-Luna et al. (2006) lo reportan, pero para el estado de Morelos como segunda especie en abundancia y mayor rango de distribución. Cortéz-Mondaca et al. (2011) hacen referencia que junto con Chrysoperla comanche (Banks) son los neurópteros con mayor presencia en cítricos del estado de Sinaloa.

Las otras especies de crisópidos identificadas con mayor frecuencia fueron C. claveri, C. cincta y Chr. externa, depredadoras que deben considerarse para investigaciones en el manejo integrado de plagas agrícolas como lo mencionan Núñez (1988a; 1988b).

El alto número de ninfas de $D$. citri consumidas por C. valida indica el potencial del insecto como enemigo natural de D. citri. Este comportamiento puede deberse a lo que menciona Chapman (1998), donde comenta que el aprovechamiento de la comida es muy variable; en algunos insectos la eficiencia de la utilización del alimento es baja, es decir, así como ingieren el alimento lo excretan, 
Tabla 2. Tablas de vida de Diaphorina citri con y sin liberación de Ceraeochrysa valida en árboles de limón mexicano bajo condiciones naturales durante los meses de diciembre 2014 y enero 2015.

Table 2. Life tables of Diaphorina citri with and without release of Ceraeochrysa valida in Mexican lemon trees under field conditions during the months of December 2014 and January 2015.

\begin{tabular}{|c|c|c|c|c|c|c|c|c|c|c|c|c|c|}
\hline \multirow[t]{4}{*}{ Estado } & \multirow{4}{*}{$\begin{array}{c}\text { Factor de } \\
\text { evaluación }\end{array}$} & \multirow{2}{*}{$\begin{array}{l}\text { No. de } \\
\text { insectos } \\
\text { (lx) }\end{array}$} & \multirow{2}{*}{$\begin{array}{l}\text { No. de } \\
\text { muertos } \\
(\mathrm{dx})\end{array}$} & \multirow{2}{*}{$\begin{array}{c}\mathrm{k}- \\
\text { value }\end{array}$} & \multicolumn{2}{|c|}{ Mortalidad } & \multirow{2}{*}{$\begin{array}{c}\% \\
\mathrm{~K}- \\
\text { value }\end{array}$} & \multirow{2}{*}{$\begin{array}{c}\text { No. de } \\
\text { insectos } \\
\text { (lx) }\end{array}$} & \multirow{2}{*}{$\begin{array}{l}\text { No. de } \\
\text { muertos } \\
(\mathrm{dx})\end{array}$} & \multirow{2}{*}{$\begin{array}{c}\mathrm{k}- \\
\text { value }\end{array}$} & \multicolumn{2}{|c|}{ Mortalidad } & \multirow{2}{*}{$\begin{array}{r}\% \\
\mathrm{~K}- \\
\text { value }\end{array}$} \\
\hline & & & & & Real & Aparente & & & & & Real & Aparente & \\
\hline & & \multicolumn{6}{|c|}{ Diciembre 2014} & \multicolumn{6}{|c|}{ Enero 2015} \\
\hline & & & & & & & a) $\mathrm{L}$ & iberación & & & & & \\
\hline \multirow{3}{*}{ Huevos } & & 215 & 185 & & 0,86 & 0,86 & & 112 & 70 & & 0,62 & 0,62 & \\
\hline & Infértiles & & 49 & 0,11 & 0,22 & 0,22 & 22,8 & & 20 & 0,08 & 0,17 & 0,17 & 17,9 \\
\hline & Depredados & & 136 & 0,74 & 0,63 & 0,63 & 82 & & 50 & 0,34 & 0,44 & 0,44 & 54,3 \\
\hline \multirow[t]{5}{*}{ Ninfas } & & 30 & 25 & & 0,11 & 0,83 & & 42 & 25 & & 0,22 & 0,59 & \\
\hline & $\begin{array}{l}\text { Liberación } \\
\text { de C. valida }\end{array}$ & & & & & & & & & & & & \\
\hline & $\begin{array}{l}\text { y otros } E \text {. } \\
\text { naturales }\end{array}$ & 25 & 0,77 & 0,11 & 0,83 & 83,3 & & 21 & 0,3 & 0,18 & 0,5 & 50 & \\
\hline & Parasitada & & 0 & 0 & 0 & 0 & & & 4 & 0,09 & 0,03 & 0,09 & 19,1 \\
\hline & Lluvia & & 0 & 0 & 0 & 0 & & & 0 & 0 & 0 & 0 & \\
\hline \multirow[t]{4}{*}{ Adulto } & & 5 & & & & & & 13 & & & & & \\
\hline & Rela. sexual & 0,5 & & & & & & 0,5 & & & & & \\
\hline & F1 progenie & 35 & & & & & & 66 & & & & & \\
\hline & & 0,16 & & & & & & 0,58 & & & & & \\
\hline \multirow[t]{2}{*}{ Total } & & & & 1,63 & 0,97 & & 97,7 & & & 0,93 & 0,84 & & 88,9 \\
\hline & & & \multicolumn{11}{|c|}{ b) Sin liberación } \\
\hline \multirow{3}{*}{ Huevos } & & 158 & 118 & & 0,74 & 0,74 & & 150 & 42 & & 0,28 & 0,28 & \\
\hline & Infértiles & & 32 & 0,09 & 0,2 & 0,20 & 20,3 & & 30 & 0,09 & 0,2 & 0,2 & 20 \\
\hline & Depredados & & 86 & 0,49 & 0,54 & 0,54 & 68,2 & & 12 & 0,04 & 0,08 & 0,08 & 10 \\
\hline \multirow[t]{4}{*}{ Ninfas } & & 40 & 16 & & 0,1 & 0,40 & & 108 & 50 & & 0,33 & 0,23 & \\
\hline & Depredación & & 15 & 0,2 & 0,09 & 0,37 & 37,5 & & 50 & 0,27 & 0,33 & 0,46 & 46,3 \\
\hline & Parasitada & & 0 & 0 & 0,00 & 0,00 & 0 & & 0 & 0 & 0 & 0 & 0 \\
\hline & Lluvia & & 0 & 0 & 0 & 0 & & & 0 & 0 & 0 & 0 & \\
\hline \multirow{4}{*}{ Adulto } & & 23 & & & & & & 32 & & & & & \\
\hline & Rela. sexual & 0,5 & & & & & & 0,50 & & & & & \\
\hline & F1 progenie & 115 & & & & & & 176 & & & & & \\
\hline & Ro & 0,72 & & & & & & 1,17 & & & & & \\
\hline Total & & & & 0,83 & 0,84 & & 85,4 & & & 0,67 & 0,44 & & 76,3 \\
\hline
\end{tabular}

aprovechando poca cantidad.

Las nueve especies de depredadores identificadas han sido reportadas en otras zonas citrícolas del país, indicando que los enemigos naturales están interactuando con D. citri y otras plagas de los cítricos (Michaud, 2004; Rodríguez-Palomera et al., 2012; Palomares-Pérez et al., 2015a).

Al observar individuos ejerciendo la depredación en campo, la reducción de ninfas en los brotes infestados con $D$. citri hace suponer que la mayor mortalidad fue ejercida durante la noche. Van Driesche et al. (2007) comentan que un cierto número de depredadores, entre ellos miembros de la familia Chrysopidae, son nocturnos. De igual forma Lundgren et al. (2010) encontraron que los porcentajes de depredación eran iguales o más altos en la noche cuando estudiaron las comunidades de depredadores de Diabrotica virgifera virgifera LeConte (Coleoptera: Chrysomelidae) en campos de maíz.

Los factores naturales, la infertilidad de los huevos, la liberación de $C$. valida y la presencia de otros enemigos naturales dieron como resultado la mayor mortalidad real en el estado de huevo, observación que coincide con Palomares-Pérez et al. (2015b) que mencionan que la tasa de mortalidad de $D$. citri se incrementa en este estado.

Otro factor importante de mortalidad a consi- 
Tabla 3. Tablas de vida de Diaphorina citri con y sin liberación de Ceraeochrysa valida en árboles de limón mexicano bajo condiciones naturales durante los meses de febrero y marzo 2015.

Table 3. Life tables of Diaphorina citri with and without release of Ceraeochrysa valida in Mexican lemon trees under field conditions during the months of February and March 2015.

\begin{tabular}{|c|c|c|c|c|c|c|c|c|c|c|c|c|c|}
\hline \multirow[t]{2}{*}{ Estado } & \multirow[t]{3}{*}{$\begin{array}{l}\text { Factor de } \\
\text { evaluación }\end{array}$} & $\begin{array}{l}\text { No. de } \\
\text { insectos } \\
\text { (lx) }\end{array}$ & $\begin{array}{l}\text { No. de } \\
\text { muertos } \\
(\mathrm{dx})\end{array}$ & $\begin{array}{c}\text { k- } \\
\text { value }\end{array}$ & $\begin{array}{r}\text { Mo } \\
\text { Real }\end{array}$ & $\begin{array}{l}\text { talidad } \\
\text { Aparente }\end{array}$ & \multirow[t]{2}{*}{$\begin{array}{c}\% \% \\
\text { K- } \\
\text { value }\end{array}$} & $\begin{array}{l}\text { No. de } \\
\text { insectos } \\
\text { (lx) }\end{array}$ & $\begin{array}{l}\text { No. de } \\
\text { muertos } \\
\text { (dx) }\end{array}$ & $\begin{array}{c}\mathrm{k}- \\
\text { value }\end{array}$ & $\begin{array}{l}\text { Mor } \\
\text { Real }\end{array}$ & $\begin{array}{l}\text { talidad } \\
\text { Aparente }\end{array}$ & $\begin{array}{c}\% \\
\% \\
\text { K- } \\
\text { value }\end{array}$ \\
\hline & & \multicolumn{5}{|c|}{ Febrero 2015} & & \multicolumn{6}{|c|}{ Marzo 2015} \\
\hline & & & & & & & a) Libe & ración & & & & & \\
\hline \multirow[t]{3}{*}{ Huevos } & & 196 & 128 & & 0,65 & 0,65 & & 154 & 40 & & 0,25 & 0,25 & \\
\hline & Infértiles & & 70 & 0,19 & 0,35 & 0,35 & 35,7 & & 30 & 0,09 & 0,19 & 0,19 & 19,5 \\
\hline & Depredados & & 58 & 0,25 & 0,29 & 0.29 & 46 & & 10 & 0,03 & 0,06 & 0,06 & 8,1 \\
\hline \multirow[t]{4}{*}{ Ninfas } & & 68 & 68 & & 0,34 & 1,00 & & 114 & 102 & & 0,66 & 0,89 & \\
\hline & $\begin{array}{l}\text { Liberación } \\
\text { de C. valida } \\
\text { y otros } \\
\text { E. naturales }\end{array}$ & 4 & 0,02 & 0,02 & 0,05 & 5,9 & & 102 & 0,97 & 0,66 & 0,89 & 89,5 & \\
\hline & Parasitada & & 0 & 0 & 0 & 0 & & & 0 & 0 & 0 & 0 & \\
\hline & Lluvia & & 63 & 1,8 & 0,32 & 0,92 & 98,4 & & & & & & \\
\hline \multirow[t]{4}{*}{ Adulto } & & 1 & & & & & & 12 & & & & & \\
\hline & Rela. Sexual & 0 & & & & & & 0,5 & & & & & \\
\hline & F1 progenie & 0 & & & & & & 75 & & & & & \\
\hline & Ro & 0 & & & & & & 0,48 & & & & & \\
\hline \multirow[t]{2}{*}{ Total } & & & & 2,29 & 1 & & 99,4 & & & 1,10 & 0,99 & & 92,2 \\
\hline & & \multicolumn{12}{|c|}{ b) Sin liberación } \\
\hline Huevos & & 260 & 210 & & 0,8 & 0,8 & & 127 & 31 & & 0,24 & 0,24 & \\
\hline & Infértiles & & 72 & 0,14 & 0,27 & 0,27 & 27,7 & & 20 & 0,07 & 0,15 & 0,15 & 15,8 \\
\hline & Depredados & & 132 & 0,52 & 0,50 & 0,50 & 70,2 & & 11 & 0,04 & 0,08 & 0,08 & 10,3 \\
\hline Ninfas & & 56 & 50 & & 0,19 & 0,89 & & 96 & 71 & & 0,55 & 0,73 & \\
\hline & Otros & & 7 & 0,05 & 0,02 & 0,12 & 12,5 & & 71 & 0,58 & 0,55 & 0,73 & 74 \\
\hline & Parasitada & & & 0,69 & 0,14 & 0,67 & 85,7 & & 0 & 0 & 0 & 0,00 & \\
\hline & Lluvia & & 38 & & & & & & & & & & \\
\hline Adulto & & 7 & & & & & & 25 & & & & & \\
\hline & Rela. sexual & 0,5 & & & & & & 0,50 & & & & & \\
\hline & F1 progenie & 32 & & & & & & 137 & & & & & \\
\hline & Ro & 0,12 & & & & & & 1,07 & & & & & \\
\hline Total & & & & 1,56 & 1,00 & & 97,3 & & & 0,70 & 0,80 & & 80,3 \\
\hline
\end{tabular}

derar es la lluvia, que se presentó únicamente en el mes de febrero y que redujo considerablemente la población de $D$. citri. Esta observación coincide con King-Cardenas, (2012) y Lomelí-Flores et al. (2009) donde mencionan que la lluvia es un factor importante en la regulación de la población de insectos.

La acción de los enemigos naturales y las condiciones naturales ejercieron una mortalidad en $D$. citri de un $23,01 \%$. Esta mortalidad se incrementó a un $73,51 \%$ deduciendo por diferencia que probablemente la liberación de $C$. valida puede llegar a ejercer hasta un control del $50 \%$. La comprensión de la interacción de los depredadores y sus fuentes de alimentación ayuda a optimizar las estrategias de control biológico (Dos Santos et al., 2016). 
Tabla 4. Mortalidad a las $24 \mathrm{~h}$ de Diaphorina citri con y sin liberación de Ceraeochrysa valida en árboles de limón mexicano en Tecomán, Colima durante los meses de diciembre de 2014 a marzo de 2015.

Table 4. Mortality at 24 hours of Diaphorina citri with and without release of Ceraeochrysa valida in Mexican lemon trees in Tecoman, Colima during the months of December of 2014 to March of 2015.

\begin{tabular}{llccc}
\hline Tratamiento & Meses & No. de individuos & No. de muertos & \% de muertos \\
\hline \multirow{5}{*}{ Liberación } & *Diciembre & 440 & 349 & 79,31 \\
& Enero & 175 & 121 & 69,14 \\
& Febrero & 145 & 97 & 66,89 \\
& Marzo & 184 & 127 & 69,09 \\
& Total & 944 & 694 & 73,51 \\
\hline \multirow{5}{*}{ Sin liberación } & *Diciembre & 200 & 50 & 25,5 \\
& Enero & 155 & 32 & 20,64 \\
& Febrero & 99 & 22 & 22,22 \\
& Marzo & 63 & 15 & 23,81 \\
& Total & 517 & 119 & 23,01 \\
\hline
\end{tabular}

*Año 2014

\section{CONCLUSIÓN}

Considerando los resultados obtenidos y tomando en cuenta que muchas de las presas de los neurópteros depredadores son plagas de importancia económica en los cultivos agrícolas, el depredador $C$. valida puede ser un agente biológico importante en el control de $D$. citri y podría formar parte del manejo integrado de esta plaga.

\section{AGRADECIMIENTOS}

Se agradece a José Luis Acevedo, propietario de la huerta, por las facilidades otorgadas para realizar los estudios de campo.

\section{LITERATURA CITADA}

Adams, P.A. 1982. Ceraeochrysa, a new genus of Chrysopinae (Neuroptera). (Studies in New World Chrysopidae, Part II). Neuroptera International 2:69-75.

Ayala-Zermeño, M.A., A. Gallou, A.M. Berlanga-Padilla, M.G. Serna-Domínguez, H.C. Arredondo-Bernal, y R.M. Montesinos-Matías. 2015. Characterisation of entomopathogenic fungi used in the biological control programme of Diaphorina citri in Mexico. Biocontrol Science and Technology 25(10):1192-1207.
Barnard, P.C. 1984. Adult morphology related to classification. p. 19-29. In M. Canard, Y. Séméria, y T.R. New. (eds.). Biology of Chrysopidae. Dr. W. Junk Publishers, The Hague, The Netherlands.

Barnett, H.L., y B.B. Hunter. 1998. Illustrated genera of imperfect fungi. 218 p. $4^{\text {th }}$ ed. The American Phytopathological Society Press, St. Paul, Minnesota, USA.

Bellows, T.S., and R.G. Van Driesche. 1999. Life table construction and analysis for evaluating biological control agents. p. 199-223. In T.S. Bellows, and T.W. Fisher (eds.). Handbook of Biological Control: Principles and Applications of Biological Control. Academic Press, San Diego, California, USA.

Brooks, S.J., y P.C. Barnard. 1990. The green lacewings of the world: a generic review (Neuroptera: Chrysopidae). Bulletin of the British Natural History Museum Entomology. 59:117-286.

Brooks, S.J. 1994. A taxonomic review of the common green lacewing genus Chrysoperla (Neuroptera: Chrysopidae). Bulletin of the British Natural History Museum Entomology 63(2):137-210.

Burks, R.A. 2003. Key to the Nearctic genera of Eulophidae, subfamilies Entedoninae, Euderinae, and Eulophinae (Hymenoptera: Chalcidoidea). Available at http//cache.ucr. edu/\%7Eheraty/Eulophidae/ (Accessed 20 April 2015). 
Cortez-Mondaca, E., J.I. López-Arroyo, L. Rodríguez-Ruíz, M.P. Partida-Valenzuela, J. Pérez-Márquez, y V.M. González-Calderón. 2011. Capacidad de depredación de especies de Chrysopidae asociadas a Diaphorina citri Kuwayama en los cítricos de Sinaloa, México. p. 323-333. En $2^{\circ}$ Simposio Nacional sobre investigación para el manejo del Psílido Asiático de los Cítricos y el Huanglongbing en México. 5 y 6 de diciembre de 2011. INIFAP, Montecillo, Texcoco, Estado de México, México.

Chapman, R.F. 1998. Alimentary canal, digestion and absorption. p. 38-66. In The insects structure and function. Cambridge University Press, Cambridge, United Kingdom.

Dos Santos, B.D.B., F.S. Ramalho, J.B. Malaquias, A.C.S. Lira, J.K.S. Pachú, F.S. Fernandes, and J.C. Zanuncio. 2016. How predation by Podisus nigrispinus is influenced by developmental stage and density of its prey Alabama argillacea. Entomologia Experimentalis et Applicata 158:142-151.

Etienne, J., D. Buckhardt, y C. Grapin. 1998. Diaphorina citri (Kuwayama) en Guadeloupe, premier signalement pour le Caraibes (Hem; Psyllidae). Bulletin de la Societé Entomologique de France 103(1):32.

Florida Preying Mantids. 2003. Florida Mantids-key to Species Diagnostic key to species of Florida Mantids Insecta-Mantodea. Available at http://entnemdept.ifas.ufl.edu/ choate/mantid_key2_03.pdf (Accessed 15 January 2015).

Gordon, R.D. 1985. The Coccinellidae (Coleoptera) of America North of Mexico. Journal of the New York Entomological Society 93(1):1912.

Halbert, S.E. y K.L. Manjunath. 2004. Asian citrus psyllids Diaphorina citri (Sternorrhyncha: Psyllidae) and greening disease in citrus: A literature review and assessment of risk in Florida. Florida Entomologist 87:330-353.

Hodges, G.S., and G.A. Evans. 2005. An identification guide to the whiteflies (Hemiptera: Aleyrodidae) of the Southeastern United States. Florida Entomologist 88(4):518-534.

Hodges, A.W., M. Rahmani, T.J. Stevens, and T.H. Spreen. 2014. Economic impacts of citrus greening (HLB) in Florida, 2012-2013. Final sponsored project report to the Florida Department of Citrus. University of Florida-IFAS, Food y Resource Economics, Gainesville, Florida. Available at www.fred.ifas.ufl.edu/ economic-impact-analysis/pdf/Economic Impacts_Florida_Citrus_Industry_2012-13. pdf (Accessed 8 May 2015).
King-Cárdenas, W.H. 2012. Dispersión de Diaphorina citri (Hemiptera: Psyllidae) en el departamento del Tolima (Colombia). Revista Tumbaga 2(7):51-60.

Lomelí-Flores, J.R., J.F. Barrera, and J.S. Bernal. 2009. Impact of natural enemies on coffee leafminer Leucoptera coffeella (Lepidoptera: Lyonetiidae) population dynamics in Chiapas, Mexico. Biological Control 51:51-60.

López-Arroyo, J.I., M. Peña, M.A. Rocha-Peña, y J. Loera. 2005. Ocurrencia en México del psílido asiático Diaphorina citri (Homoptera: Psyllidae). p. C68. En Memorias del VII Congreso Internacional de Fitopatología. 26 al 29 de septiembre de 2005. Sociedad Mexicana de Fitopatología, Chihuahua, México.

Lundgren, J.G., S. Toepfer, T. Haye, y U. Kuhlmann. 2010. Haemolymph defense of an invasive herbivore: its breadth of effectiveness against predators. Journal of Applied Entomology 134(5):439-448.

Michaud, J.P. 2004. Natural mortality of Asian citrus psyllid (Homoptera: Psyllidae) in central Florida. Biological Control 29(2):260-269.

Núñez, Z.E. 1988a. Chrysopidae (Neuroptera) del Perú y sus especies más comunes. Revista Peruana de Entomología 31:69-75.

Núñez, Z.E. 1988b. Ciclo biológico y crianza de Chrysoperla externa y Ceraerochrysa cincta (Neuroptera: Chrysopidae). Revista Peruana de Entomología 31:76-82.

Palomares-Pérez, M., J.M. Rodríguez-Vélez, B. Rodríguez-Vélez, A. Marín-Jarillo, J.A. SánchezGonzález, and H.C. Arredondo-Bernal. 2015a. First record and predatory activity of Exochomus marginipennis (LeConte) (Coleoptera: Coccinellidae) on Diaphorina citri Kuwayama (Hemiptera: Liviidae). Entomological News 125(3):194-198.

Palomares-Pérez, M., E.G. Córdoba-Urtiz, J.A. Sánchez-González, N.I. Medina-García, R. Hernández-Mendoza, V.H. Pérez-Díaz y H.C. Arredondo-Bernal. 2015b. Aspectos biológicos de Diaphorina citri (Hemiptera: Liviidae) desarrollada en invernadero bajo condiciones no controladas. Revista Colombiana de Entomología 41(2):228-234.

Peña-Martínez, R., N. Villegas-Jiménez, y J.R. Lomelí-Flores. 2002. Identificación de pulgones (Homoptera: Aphididae) con énfasis en el pulgón café de los cítricos Toxoptera citricida (Kirkaldy). p. 53-68. En H.C. Arredondo-Bernal, M.A. Mellin-Rosas, P. Pérez-Serrato, y J.P. Martínez-Soriano (eds.). Control biológico del pulgón café Toxoptera citricida, vector del virus de la tristeza de los cítricos.. Subdirección de Control Biológico, Centro Nacional de Referencia Fitosanitaria, SAGARPA, México. 
Petrakis, P.V., and P. Moulet. 2011. First record of the nearctic Zelus renardii (Heteroptera, Reduviidae, Harpactocorinae) in Europe. Entomologia Hellenica 20:75-81.

Rodríguez-Palomera, M., J. Cambero-Campos, A. Robles-Bermúdez, C. Carvajal-Cazola, y O. Estrada-Virgen. 2012. Enemigos naturales asociados a Diaphorina citri kuwayama (Hemiptera: Psyllidae) en limón persa (Citrus latifolia Tanaka) en Nayarit, México. Acta Zoológica Mexicana (nueva serie) 28(3):625-629.

SAGARPA. 2009. Norma Oficial Mexicana de Emergencia NOM-EM-047-FITO-2009. Secretaria de Agricultura, Ganadería, Desarrollo Rural, Pesca y Alimentación. Diario Oficial de la Federación (DOF), 8 de junio de 2009, Ciudad de México, México.

SAS. 2008. SAS Users Guide: Statistics Version 9.2 for Windows. SAS Institute Inc., Cary, North Carolina, USA.

SCOPE. 2015. Sistema Coordinado para la Vigilancia de Plagas Reglamentadas y su Epidemiología. Disponible en http://www.scopemex.uaslp.mx (Consulta 15 abril 2015

SINAVEF. 2013. Sistema Nacional de Vigilancia Epidemiológica Fitosanitaria. Reporte Epidemiológico Huanglongbing (HLB). Disponible en http://langif.uaslp.mx/documentos/ privada/BoletinesVarios/HLB/001.pdf (Consulta 20 de mayo de 2015).

Tauber, C.A., and T. De León. 2001. Systematics of green lacewings (Neuroptera: Chrysopidae): Larvae of Ceraeochrysa from Mexico. Annals of the Entomological Society of America 94(2):197-209.

Tauber, C.A., T. de León, N.D. Penny, and M.J. Tauber. 2000. The genus Ceraeochrysa (Neuroptera: Chrysopidae) of America North of Mexico: Larvae, Adults, and Comparative Biology. Annals of the Entomological Society of America 93(6):1195-1221.
Thomas, D.B. 2002. Trip report: status of the brown citrus aphid in the Mexican state of Campeche: April 2002. p. 9. USDA-ARS. Kika de la Garza Subtropical Agriculture Research Center, Weslaco, Texas, USA.

Tjeder, B. 1966. Neuroptera- Planipennia. Family Chrysopidae. South African Animal Life 12:238-534.

Tjeder, B. 1971. Redescriptions lectotype designations of some exotic Chrysopidae (Neur.) Entomologica Scandinavica 2:110-188.

Tsai, J., J. Wang, y Y. Liu. 2002. Seasonal abundance of the asian citrus psyllid, Diaphorina citri (Homoptera: Psyllidae) in Southern Florida. Florida Entomologist 85(3):446-451.

Valencia-Luna, L.A., J. Romero-Nápoles, J. Valdez-Carrasco, J.L. Carrillo-Sánchez, y V. López-Martínez. 2006. Taxonomía y registros de Chrysopidae (Insecta: Neuroptera) en el estado de Morelos, México. Acta Zoológica Mexicana (nueva serie) 22(1):17-61.

Van Driesche, R.G., M.S. Hoddle, y T.D. Center. 2007. Control de plagas y malezas por enemigos naturales. p. 43. US Department of Agriculture, Forest Health Technology Enterprise Team, Washington D.C., USA.

Weirauch, Ch., C. Alvarez, and G. Zhang. 2012. Zelus renardii and Z. tetracanthus (Hemiptera: Reduviidae): biological attributes and the potential for dispersal in two assassin bug species. Florida Entomologist 95(3):641-649. 